\title{
Initiating droxidopa for neurogenic orthostatic hypotension in a patient with Parkinson disease
}

\author{
Daniel Claassen ${ }^{1} \cdot$ Mark Lew $^{2}$
}

Received: 7 June 2017/ Accepted: 7 June 2017/Published online: 19 June 2017

(c) The Author(s) 2017. This article is an open access publication

Keywords Droxidopa $\cdot$ Neurogenic orthostatic hypotension · Parkinson's disease

\section{Challenge questions}

What is the rationale for choosing pharmacologic therapy for patients with neurogenic orthostatic hypotension? What are the criteria to start with droxidopa?

\section{Case presentation}

Mr. F is a 64-year-old male with Parkinson disease (PD) who presents with fatigue, urinary urgency and frequency, and, more recently, difficulty working as a part-time tollbooth operator. His motor symptoms related to PD have been present for about 7 years, and he has been maintained on carbidopa/ levodopa $25 \mathrm{mg} / 100 \mathrm{mg}$ three times daily (TID) and ropinirole $3 \mathrm{mg}$ TID until now. For the last 6 months, the patient reports a "wobbly or dizzy sensation when standing." Four months ago, he was seen by his family physician who noted that he had a blood pressure (BP) drop upon standing. The family physician instructed the patient to liberalize fluid and salt intake and gave the patient a prescription for knee-high compression garments.

As part of his current routine, the patient takes a brief nap in the afternoon for $45 \mathrm{~min}$ after his lunch when he is off

Daniel Claassen

daniel.claassen@vanderbilt.edu

1 Department of Neurology, Vanderbilt University, Nashville, TN, USA

2 Department of Neurology, University of Southern California, Los Angeles, CA, USA from his job and at home. At work, he reports that he has become more fatigued and that colleagues have noticed that he processes thoughts more slowly. In fact, the patient reports mild memory problems, which became more marked when his family physician prescribed him the anticholinergic drug tolterodine for urinary frequency and urgency.

At a visit to his movement disorder neurologist 3 months ago, his BP was 98/75 $\mathrm{mmHg}$ sitting and $75 / 60 \mathrm{mmHg}$ upon standing with little increase in heart rate (HR). He reported feeling lightheaded and about to faint upon standing. He also reported fatigue, urinary symptoms, and memory complaints, all common in patients with $\mathrm{PD}$ and neurogenic $\mathrm{OH}$ $(\mathrm{nOH})$. At this visit, his medications were reviewed and adjusted. Treatment of his urinary symptoms with an anticholinergic medication most likely exacerbated his memory problems and daytime sleepiness. Therefore, tolterodine was discontinued. Because dopamine agonists have been associated with low BP, dopamine agonists were decreased to pramipexole dihydrochloride $1 \mathrm{mg}$ at bedtime.

A few weeks after visiting his neurologist, he was placed on medical disability and stopped going to work. His daytime naps increased to twice daily, and he continued to complain about feeling "lightheaded" in the morning and upon standing.

At his next visit to his neurologist, the patient reported that his napping has become more frequent, and he continued to have burdensome symptoms of $\mathrm{nOH}$, which did not remit after his dopaminergic medications were reduced.

\section{Expert commentary (Dr. Claassen)}

Neurogenic $\mathrm{OH}$ is a manifestation of autonomic failure, which is characterized by inappropriate norepinephrine (NE) release from sympathetic post-ganglionic nerves 
when standing. Starting this patient on droxidopa, which is a synthetic precursor of $\mathrm{NE}[1,4]$, is a reasonable option at this stage. Additionally, given that this patient needs to nap several times daily, there is a mechanistic hypothesis that increasing NE levels in the CNS may potentially improve other non-motor symptoms in PD [2], although no large studies have been performed to confirm this.

\section{Case continuation}

The patient was placed on droxidopa $100 \mathrm{mg}$ on a modified TID schedule (doses taken before arising from bed in the morning, at lunchtime, and at least 3-4 h before bedtime) and instructed to increase the dose by $100 \mathrm{mg}$ on this modified TID schedule every 24-48 h until remission of $\mathrm{nOH}$ symptoms or up to a maximum droxidopa dosage of $600 \mathrm{mg}$ TID. To make sure that the patient did not develop supine hypertension (sHTN), which is a frequent finding in patients with $\mathrm{nOH}[3,5]$, he was instructed to monitor his $\mathrm{BP}$ in the supine and standing (3 min after) positions first thing in the morning during the titration of droxidopa. The patient was also counseled to avoid the supine position during the daytime, to take naps in a recliner chair, and to elevate the head of his bed by $30^{\circ}-45^{\circ}$ at bedtime.

After achieving a droxidopa dose of $500 \mathrm{mg}$ on the modified TID schedule, the patient reported that his $\mathrm{nOH}$ symptoms had resolved. This patient has continued this dosage of droxidopa for 6 months and has experienced many fewer nOH symptoms with no adverse effects to date.

\section{Expert commentary (Dr. Lew)}

It is important to carefully consider the risk of sHTN in patients taking medications to increase BP, including droxidopa, midodrine, or fludrocortisone. In this regard, this patient is particularly complicated because he naps several times daily. He needed to be reminded to elevate the head of his bed at least $30^{\circ}-45^{\circ}$ at bedtime, to take his naps in a recliner chair, and to attempt to dose his droxidopa after awakening from his naps, if possible. Additionally, careful review of concomitant medications prescribed by other specialists is crucial. In this case, tolterodine, an anticholinergic agent to treat bladder dysfunction, likely caused and/or contributed to the cognitive impairment.

\section{Compliance with ethical standards}

Conflict of interest Dr. Claassen has received research grants from NIH/NINDS, Michael J. Fox Foundation, Vaccinex, CHDI, C2 N, and Auspex, and he is a consultant for Teva Neuroscience, Lundbeck, Acadia, and AbbVie. Dr. Lew is a consultant for Lundbeck.

Funding source This manuscript is part of a supplement sponsored by Lundbeck.

Open Access This article is distributed under the terms of the Creative Commons Attribution 4.0 International License (http://crea tivecommons.org/licenses/by/4.0/), which permits unrestricted use, distribution, and reproduction in any medium, provided you give appropriate credit to the original author(s) and the source, provide a link to the Creative Commons license, and indicate if changes were made.

\section{References}

1. Elgebaly A, Abdelazeim B, Mattar O, Gadelkarim M, Salah R, Negida A (2016) Meta-analysis of the safety and efficacy of droxidopa for neurogenic orthostatic hypotension. Clin Auton Res 26:171-180

2. Espay AJ, LeWitt PA, Kaufmann H (2014) Norepinephrine deficiency in Parkinson's disease: the case for noradrenergic enhancement. Mov Disord 29:1710-1719

3. Fanciulli A, Gobel G, Ndayisaba JP, Granata R, Duerr S, Strano S, Colosimo C, Poewe W, Pontieri FE, Wenning GK (2016) Supine hypertension in Parkinson's disease and multiple system atrophy. Clin Auton Res 26:97-105

4. Kaufmann H, Norcliffe-Kaufmann L, Palma JA (2015) Droxidopa in neurogenic orthostatic hypotension. Expert Rev Cardiovasc Ther 13:875-891

5. Umehara T, Matsuno H, Toyoda C, Oka H (2016) Clinical characteristics of supine hypertension in de novo Parkinson disease. Clin Auton Res 26:15-21 